\title{
Novel Gene Variants in SRY-negative 46, XX male syndrome with Bone Marrow Failure by Whole Exome Sequencing
}

\author{
Aoli Zhang ${ }^{1}$, Li-Xian Chang ${ }^{1}$, Li Zhang ${ }^{1}$, Chao Liu ${ }^{1}$, Li-Peng Liu ${ }^{1}$, xiaoyan Chen ${ }^{1}$, Meihui \\ $\mathrm{Yi}^{1}$, Yang Lan ${ }^{1}$, Luyang Zhang ${ }^{1}$, Yu-Li Cai ${ }^{1}$, jing feng ${ }^{1}$, Wenqi Wu ${ }^{1}$, Yingchi Zhang ${ }^{1}$, and \\ Xiaofan $\mathrm{Zhu}^{2}$ \\ ${ }^{1}$ Chinese Academy of Medical Sciences Institute of Hematology and Blood Diseases \\ Hospital \\ ${ }^{2}$ Institute of Hematology \& Blood Diseases Hospital Chinese Academy of Medical Sciences \\ \& PeKing Union Medical College
}

June 12,2020

\begin{abstract}
46, XX male syndrome is a rare disorder of sex development. One-tenth of 46, XX male syndrome is sex-determining region Y (SRY)-negative. We used whole-exome sequencing (WES) analysis associated genes to investigate the underlying genetic etiology of 46, XX male syndrome patients with bone marrow failure with a typical male phenotype. WES reveals SRY and SRY-box family genes were negative. Simultaneously, gene variants were detected in female pathway, testis development, and steroid receptor genes. There are undefined gene variants associated with congenital bone marrow failure. WES proved an efficient diagnostic method toward 46, XX male syndrome patients with hematological disorder.
\end{abstract}

\section{INTRODUCTION}

46, XX male syndrome is a rare disorder of sex development(DSD) characterized by a female karyotype in conformity with a male phenotype ${ }^{(1)}$, which had been reported in 1964 firstly $^{(2)}$. The prevalence of diagnosed 46, XX male syndrome was approximately 4 per 100000 newborn males ${ }^{(3,4)}$. 46, XX males exist three different clinical categories with typical XX males, genital ambiguities, and real hermaphrodites. It had reported 46, XX male syndrome with chronic autoimmune thyroiditis ${ }^{(5)}$, and with myelodysplastic syndrome converted to acute megakaryocyte leukemia ${ }^{(6)}$. We herein report a case of sex-determining region Y(SRY)-negative 46, XX male syndrome co-existing with bone marrow failure in a child with a typical male phenotype.

Whole exome sequencing (WES) reveals SRY and SRY-box family genes was negative in this patient. Gene variants were detected by WES, which associated with the loss-of-function in the female pathway, testis development, and some steroid receptor genes of sex differentiation These variants could affect gonad development with a typical male phenotype.

\section{Case presentation}

In 2017 May, an 8-year-old male referred to hospital with pancytopenia and epistaxis. Bone marrow smears indicated reduced bone marrow hyperplasia. Bone marrow biopsy showed hypoplastic hematopoietic without megakaryocytes. Paroxysmal nocturnal hemoglobinuria clone was negative. His diagnosis considered as aplastic anemia in the local hospital. He had taken dexamethasone, stanozolol, and ciclosporin. The response to the treatment is well, whereas platelet count was $23-59 \times 10^{9} / \mathrm{L}$. 
In June 2018, he admitted to our department with a complaint of platelet count dropped to $10 \times 10^{9} / \mathrm{L}$ with epistaxis intermittently. He had skin ecchymosis without splenomegaly and hepatomegaly. The male was born by a G3P2A1 mother who had premature delivery by cesarean section for abdominal pain by trauma. The cause of abortion was unknown, and his sister is healthy. Birth weight is $2.4 \mathrm{~kg}$. His body weight and height were $31 \mathrm{~kg}$ and $135 \mathrm{~cm}$, respectively. Physical examination showed small head, small eye crack, right thumb evagination, bipedal walking inside eight-character, without milk coffee spot. Soft and normal testis were palpated.

Color doppler ultrasonography showed a normal male reproductive system. Testosterone concentration was in normal ranges. Virus detection and rheumatism inspection were negative. Repeated bone marrow smears showed hypoplastic of marrow. Trephine biopsy indicated hyperplasia of marrow diminished severely and lack of hemocyte without signs of marrow fibrosis. Bone marrow and peripheral blood culture revealed 46 chromosomes with XX constitution. Gene mutations, fusion genes, fluorescence in situ hybridization, and comet assay were no definite positive result.

\section{MATERIALS AND METHODS}

\section{Genetic Analysis}

The genomic DNA was extracted by Universal Genomic DNA kit (CWBIO\# CWS002). And the exome capture, high throughput sequencing, and standard filtering were performed in the Beijing Novogene Technology Co., Ltd. (Beijing, China, http://www.novogene.com). The genomic DNA samples were randomly fragmented approximately 180 to 280 bp long by Covaris. All exomes were captured by Agilent SureSelect Human All Exon V6 kit and sequenced by Illumina HiSeq Pair End 150 bp platform. The available sequencing data were compared with the reference genome (GRCh37/hg19). The bioinformatics analyses were conducted by Novogene and Mygenostics in Beijing, China. WES indicated gene variants associated with DSD, such as structural variation (SV), single-nucleotide variation (SNV), and insertion and deletions (INDELs).

\section{RESULTS}

\section{Whole-exome sequencing}

WES reveals the SRY gene is negative. SV in loss-of-function in the female pathway: WNT4, RSPO1, CTNNB1, and INSRR. Gene variants were detected associated with 46, XX disorder of testis development: DMRT1, and FOG2/ZFPM2. Variant steroid receptor genes: CYP17A1, STAR, SRD5A2, and AR (Table 1, Table S1and Figure 1). There are undefined gene variants associated with congenital bone marrow failure.

\section{Diagnosis and treatment}

The patient was diagnosed with the SRY-negative 46, XX male syndrome co-existing with bone marrow failure. He took ciclosporin 50mg q12h, stanozolol 2mg QD, and levamisole 50mg QD. The complete blood count remains stable.

\section{DISCUSSION}

46, XX male syndrome is a rare sex reversal syndrome with a frequency of 1 in 20,000-25,000 newborn males ${ }^{(3,4)}$. Phenotypically, three clinical categories of 46 , XX male syndrome individuals have been identified $^{(7)}$. The classic form of $46, \mathrm{XX}$ male syndrome is testicular DSD, which has a female karyotype and phenotypically male. We herein report a classical form of the SRY-negative 46, XX male syndrome co-existing with bone marrow failure.

Broadly, these conditions are caused by gain-of-function variants in crucial testis differentiation genes or loss-of-function variants in ovarian differentiation genes ${ }^{(8)} .10 \%$ of testicular DSD is SRY-negative. In the absence of SRY, there was overexpression of other pro-testis genes, e.g. SRY-box (SOX) family genes, or failure of pro-ovarian/anti-testis genes, e.g. WNT4, and RSPO1 ${ }^{(9)}$. WES reveals the SRY gene was negative in this patient. At the same time, the patient harbored variants gene associated with SRY-negative 46, XX 
male syndrome. Testicles development is a large and complex network. This patient had multiple genetic mutations associated with testicles development.

Few genes have been implicated in ovarian development compared with testicular development relatively. In humans, patients carrying a heterozygous mutation of the WNT4 gene present Müllerian duct abnormalities and hyperandrogenism ${ }^{(10)}$. Homozygous missense mutation WNT4 gene is a disease-causing in SERKAL syndrome $^{(11)}$. Mutations in the RSPO1 gene have been associated with the SRY gene-negative 46, XX testicular DSD, as well as with ovo-testicular DSD ${ }^{(12)}$. WNT4 and RSPO1 activate canonical WNT signaling, and CTNNB1 is a crucial regulatory molecule of the Wnt signaling pathway. Indeed, several feedback loops help maintain WNT4/RSPO1 signaling, including the insulin signaling pathway (consists of INSR and INSRR). Albeit SRY gene and SOX9 gene are absent in this patient, SV of WNT4, RSPO1, and STNNB1 could result in loss-of-function of the central female pathway, which leads to female-to-male sex reversal of the gonads. Besides, there was a large fragment inserted in INSRR affecting positive feedback loop.

DMRT1 activates testis-promoting genes including Sox9, Sox8 and PTGDS, and represses ovary-promoting genes such as Wnt4 and Rspo1 ${ }^{(13)}$. DMRT1 mutations in the case may activate testis-promoting genes and represses ovary-promoting genes. Independent missense mutations of FOG2 gene associated with 46, XY DSD in human. Further studies indicated impaired the ability of FOG2 proteins interact with GATA4, an early testicular development regulator ${ }^{(14)}$. Large fragment deletion in FOG2 had not reported in 46, XX DSD, we should force further theorization to the mechanism.

Impaired activity of P450 oxidoreductase gene-dependent microsomal enzymes can lead to DSD, pubertal failure, adrenal dysfunction, and maternal virilization during pregnancy ${ }^{(15)}$. Large fragment deletion CYP17A1 may be other molecular mechanisms to cause 46, XX DSD. STAR involved in male sex development plays a critical role in testosterone biosynthesis. STAR gene mutations lead to blockage of the synthesis of testosterone and other steroids. It was reported that dual gene defects (p. A596T in AR and p. G196S in SRD5A2) in a patient with hypospadias ${ }^{(16)}$. Interestingly, in contrast to patients carrying novel gene variants in STAR, SRD5A2 and AR, the patient has typical genitalia. During puberty, the patient may present gynecomastia, and/or adrenal failure in late adolescence or adulthood.

We describe a rare case of SRY-negative 46, XX male syndrome co-existing with bone marrow failure. WES reveals SRY gene and SRY-box family genes were negative. Gene variants could affect testicles development of $46 \mathrm{XX}$ male syndrome. WES is more comprehensive in detecting genetic mutations of SDS. The underlying mechanisms of SRY negative 46, XX males with bone marrow failure needs to be further studied.

\section{CONFLICTS OF INTEREST}

The authors have no conflicts of interest relevant to this article to disclose.

\section{ACKNOWLEDGMENTS}

We would like to thank the patient and his families for participation in this study. This work was supported by AiYou Foundation and the National Key Research and Development Program of China (2016YFC0901503). We would like to acknowledge Mygenostics for their technical assistance.

\section{ETHICS}

The Ethics committee of the Institute of Hematology and Blood Diseases Hospital, Chinese Academy of Medical Sciences and Peking Union Medical College approved.

\section{INFORMED CONSENT}

Parental of the patient consent obtained. 


\section{DATA AVAILABILITY STATEMENT}

The data that support the findings of this study are available from the corresponding author upon reasonable request.

\section{REFERENECES}

1. Ergun-Longmire B, Vinci G, Alonso L, Matthew S, Tansil S, Lin-Su K, et al. Clinical, hormonal and cytogenetic evaluation of 46,XX males and review of the literature. J Pediatri Endocrinol Metab.2005;18(8):739-748.

2. Delachapelle A, Hortling H, Niemi M, Wennstroem J. XX SEX CHROMOSOMES IN A HUMAN MALE. FIRST CASE. Acta Med Scandi . 1964;175:Suppl 412:25-28.

3. de la Chapelle A. The etiology of maleness in XX men. Hum Genet. 1981;58(1):105-116.

4. Berglund A, Johannsen TH, Stochholm K, Aksglaede L, Fedder J, Viuff MH, et al. Incidence, prevalence, diagnostic delay, morbidity, mortality and socioeconomic status in males with 46,XX disorders of sex development: a nationwide study. Hum Reprod.2017;32(8):1751-1760.

5. Mustafa O, Mehmet E. A 46, XX SRY - negative man with infertility, and co-existing with chronic autoimmune thyroiditis. Gynecol Endocrinol. 2010;26(6):413-5.

6. Lau LC, Lim P, Lee LH, Hwang W, Lui WO, Chong YY. Myelodysplastic syndrome with transformation to AML-M7 in a 46,XX male patient.Cancer Genet Cytogenet. 2002;136(2):153-154.

7. Abbas NE, Toublanc JE, Boucekkine C, Toublanc M, Affara NA, Job JC, et al. A possible common origin of "Y-negative" human XX males and XX true hermaphrodites. Hum Genet . 1990;84(4):356-360.

8. Knarston I, Ayers K, Sinclair A. Molecular mechanisms associated with 46,XX disorders of sex development. Clin Sci.2016;130(6):421-432.

9. Grinspon RP, Rey RA. Disorders of Sex Development with Testicular Differentiation in SRY-Negative 46,XX Individuals: Clinical and Genetic Aspects. Sex Dev. 2016;10(1):1-11.

10. Philibert P, Biason-Lauber A, Rouzier R, Pienkowski C, Paris F, Konrad D, et al. Identification and functional analysis of a new WNT4 gene mutation among 28 adolescent girls with primary amenorrhea and mullerian duct abnormalities: a French collaborative study. J Clin Endocrinol Metab. 2008;93(3):895-900.

11. Mandel H, Shemer R, Borochowitz ZU, Okopnik M, Knopf C, Indelman M, et al. SERKAL syndrome: an autosomal-recessive disorder caused by a loss-of-function mutation in WNT4. Am J Hum Genet.2008;82(1):39-47.

12. Tomaselli S, Megiorni F, De Bernardo C, Felici A, Marrocco G, Maggiulli G, et al. Syndromic true hermaphroditism due to an R-spondin1 (RSPO1) homozygous mutation. Hum Mutat. 2008;29(2):220-226.

13. Lindeman RE, Gearhart MD, Minkina A, Krentz AD, Bardwell VJ, Zarkower D. Sexual cell-fate reprogramming in the ovary by DMRT1. Curr Biol. 2015;25(6):764-771.

14. Bashamboo A, Brauner R, Bignon-Topalovic J, Lortat-Jacob S, Karageorgou V, Lourenco D, et al. Mutations in the FOG2/ZFPM2 gene are associated with anomalies of human testis determination. Hum Mol Genet. 2014;23(14):3657-3665.

15. Fukami M, Ogata T. Cytochrome P450 oxidoreductase deficiency: rare congenital disorder leading to skeletal malformations and steroidogenic defects. Pediatr Int.2014;56(6):805-808.

16. Nagaraja MR, Gubbala SP, Delphine Silvia CRW, Amanchy R. Molecular diagnostics of disorders of sexual development: an Indian survey and systems biology perspective. Syst Biol Reprod Medicine . 2019;65(2):105-120.

FIGURE 1 Whole exome sequencing reveals gene variants in SRY negative 46, XX males with bone marrow failure. 


\section{Hosted file}

TABLE 1.docx available at https://authorea.com/users/332735/articles/459153-novel-genevariants-in-sry-negative-46-xx-male-syndrome-with-bone-marrow-failure-by-whole-exomesequencing
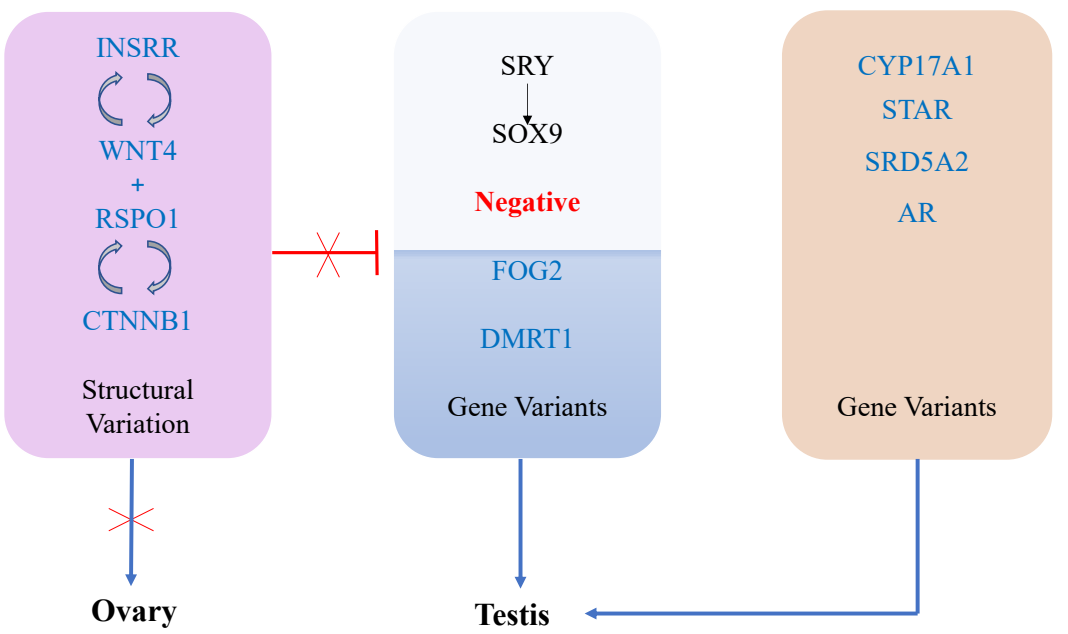